Swarthmore College

Works

2-1-2016

\title{
A Disk-Based Dynamical Constraint On The Mass Of The Young Binary DQ Tau
}

I. Czekala

S. M. Andrews

G. Torres

Eric L.N. Jensen

Swarthmore College, ejensen1@swarthmore.edu

K. G. Stassun

See next page for additional authors

Follow this and additional works at: https://works.swarthmore.edu/fac-physics

Part of the Astrophysics and Astronomy Commons

Let us know how access to these works benefits you

\section{Recommended Citation}

I. Czekala, S. M. Andrews, G. Torres, Eric L.N. Jensen, K. G. Stassun, D. J. Wilner, and D. W. Latham. (2016). "A Disk-Based Dynamical Constraint On The Mass Of The Young Binary DQ Tau". Astrophysical Journal. Volume 818, Issue 2. DOI: 10.3847/0004-637X/818/2/156

https://works.swarthmore.edu/fac-physics/253

This work is brought to you for free by Swarthmore College Libraries' Works. It has been accepted for inclusion in Physics \& Astronomy Faculty Works by an authorized administrator of Works. For more information, please contact myworks@swarthmore.edu. 


\section{Authors}

I. Czekala, S. M. Andrews, G. Torres, Eric L.N. Jensen, K. G. Stassun, D. J. Wilner, and D. W. Latham 


\title{
A DISK-BASED DYNAMICAL CONSTRAINT ON THE MASS OF THE YOUNG BINARY DQ TAU
}

\author{
I. Czekala ${ }^{1}$, S. M. Andrews ${ }^{1}$, G. Torres ${ }^{1}$, E. L. N. Jensen ${ }^{2}$, K. G. Stassun ${ }^{3,4}$, D. J. Wilner ${ }^{1}$, and D. W. Latham ${ }^{1}$ \\ ${ }^{1}$ Harvard-Smithsonian Center for Astrophysics, 60 Garden Street, Cambridge, MA 02138, USA; iczekala@cfa.harvard.edu \\ ${ }^{2}$ Department of Physics and Astronomy, Swarthmore College, 500 College Avenue, Swarthmore, PA 19081, USA \\ ${ }^{3}$ Department of Physics and Astronomy, Vanderbilt University, Nashville, TN 37235, USA \\ ${ }^{4}$ Department of Physics, Fisk University, Nashville, TN 37208, USA \\ Received 2015 December 1; accepted 2016 January 14; published 2016 February 17
}

\begin{abstract}
We present new Atacama Large Millimeter/Submillimeter Array (ALMA) observations of CO $J=2-1$ line emission from the DQ Tau circumbinary disk. These data are used to tomographically reconstruct the Keplerian disk velocity field in a forward-modeling inference framework, and thereby provide a dynamical constraint on the mass of the DQ Tau binary of $M_{*}=1.27_{-0.27}^{+0.46} M_{\odot}$. Those results are compared with an updated and improved orbital solution for this double-lined system based on long-term monitoring of its stellar radial velocities. Both of these independent dynamical constraints on the binary mass are in excellent agreement: taken together, they demonstrate that the DQ Tau system mass is $1.21 \pm 0.26 M_{\odot}$ and that the disk and binary orbital planes are aligned within $3^{\circ}$ (at $3 \sigma$ confidence). The predictions of various theoretical models for pre-main-sequence stellar evolution are also consistent with these masses, though more detailed comparisons are difficult due to lingering uncertainties regarding the photospheric properties of the individual components. DQ Tau is the third, nearly equal-mass, double-lined spectroscopic binary with a circumbinary disk that has been dynamically "weighed" with these two independent techniques: all show consistent results, validating the overall accuracy of the disk-based approach and demonstrating that it can be robustly applied to large samples of young, single stars as ALMA ramps up to operations at full capacity.
\end{abstract}

Key words: protoplanetary disks - stars: fundamental parameters - stars: individual (DQ Tau) - stars: pre-main sequence

\section{INTRODUCTION}

Theoretical models for pre-main-sequence (pre-MS) stellar evolution are fundamental tools for learning about star and planet formation. However, the accuracy of such modelsespecially at young ages-is unclear, due to our limited understanding of some complex physical effects, such as accretion (e.g., Baraffe et al. 2009) or magnetic fields and convection (e.g., Feiden \& Chaboyer 2013). While such issues are being explored theoretically, robust observational constraints on key stellar parameters can be used to help guide improvements to the models. Most important are dynamical constraints on stellar masses, $M_{*}$ (e.g., see Hillenbrand \& White 2004). Usually these are determined from the orbital motions of binary systems (Stassun et al. 2014), but they could increasingly be measured for single stars based on the rotation of their associated gas disks (e.g., Simon et al. 2000).

We are interested in comparing the constraints of these two approaches to illuminate and quantify any associated systematic problems in the inference of $M_{*}$. To do that, we have targeted the few roughly equal-mass double-lined spectroscopic binaries that host circumbinary disks, including V4046 Sgr (Rosenfeld et al. 2012) and AK Sco (Czekala et al. 2015). In both cases, excellent agreement (to within $\sim 1 \%$ ) is found between the estimates of $M_{*}$ from radial-velocity (RV) monitoring of the stars and the tomographic reconstruction of the $\mathrm{CO}$ gas velocity field in the disk. The confluence of these measurements also indicates that the disk and binary orbital planes are well-aligned (within $1^{\circ}-2^{\circ}$ ). Moreover, the predictions of theoretical pre-MS models faithfully reproduce these results for these two particular examples. However, these model successes are perhaps not surprising, since both V4046 Sgr and AK Sco are relatively old (10 and $18 \mathrm{Myr}$ ) and massive
(1.8 and $2.5 M_{\odot}$ in each system) and the models should be more robust in that range of age and mass. An important supplementary test would employ a cooler and younger binary.

In those respects, DQ Tau is an exemplary target. DQ Tau is a roughly equal-mass double-lined spectroscopic binary with a period of $\sim 16$ days and a notably eccentric orbit (Mathieu et al. 1997). It has a composite spectral type of $\sim$ M0-M1 (e.g., Herbig 1977; Herczeg \& Hillenbrand 2014) and is located in the nearby and relatively young Taurus clouds. DQ Tau exhibits enhancements of various tracers of accretion and activity_optical brightening (Mathieu et al. 1997), emission line variations (Basri et al. 1997), and millimeter/radio emission (Salter et al. 2010) - that have been associated with both pulsed accretion and reconnection events from colliding magnetospheres near periastron. The DQ Tau binary hosts a circumbinary disk with substantial millimeter continuum emission from dust (Beckwith et al. 1990; Andrews \& Williams 2005; Guilloteau et al. 2011). There is recent evidence for molecular gas in rotation around the central binary host (Williams \& Best 2014), though there is nonnegligible contamination from the local molecular cloud (Guilloteau et al. 2013).

We present new observations of molecular gas in the DQ Tau circumbinary disk made with the Atacama Large Millimeter/Submillimeter Array (ALMA), and use them to place a dynamical constraint on the total mass of the DQ Tau binary. We also provide an updated orbital solution for the DQ Tau binary based on long-term RV monitoring. Section 2 presents the data and their calibration. Section 3 describes our modeling of the gas disk velocity field, provides an update of the original Mathieu et al. (1997) orbital solution, and highlights the key results. Section 4 discusses these results together and assesses the predictions of pre-MS evolution 
models. Furthermore, Section 5 provides a summary in the context of other young circumbinary disk systems and the utility of the disk-based dynamical mass technique.

\section{OBSERVATIONS AND DATA REDUCTION}

\subsection{Millimeter Interferometry}

ALMA observed the DQ Tau system on 2015 May 24, using 34 of its $12 \mathrm{~m}$ antennas with separations ranging from 21 to $540 \mathrm{~m}$. The observations were configured with the same spectral setup as in Czekala et al. (2015), employing the Band 6 receivers to cover the $\mathrm{CO},{ }^{13} \mathrm{CO}$, and $\mathrm{C}^{18} \mathrm{O} J=2-1$ transitions in 61, 61, and $122 \mathrm{kHz}$ channels, respectively, as well as the adjacent continuum (at $232 \mathrm{GHz}$, or $1.3 \mathrm{~mm}$ ). The nearby quasar $\mathrm{J} 0510+1800\left(6^{\circ}\right.$ separation $)$ was observed regularly to monitor variations in the complex gain response of the interferometer. The bright quasar J0423-0120 was also observed to calibrate the bandpass behavior and absolute flux levels. The total on-source integration time was $\sim 28$ minutes. The visibilities were calibrated using standard techniques with the CASA software package (v4.3). After a phase and amplitude self-calibration based on the bright continuum, the spectral line visibilities were time-averaged (to $30 \mathrm{~s}$ intervals) and continuum-subtracted.

Images of the continuum and spectral line data were created by Fourier inverting the calibrated visibilities (assuming a Briggs robust weighting parameter of 0.5 , to balance the signalto-noise ratio $(\mathrm{S} / \mathrm{N})$ and resolution), deconvolving with the CLEAN algorithm, and restoring with a synthesized beam with FWHM $=0$ ". $8 \times 00^{\prime \prime} 6\left(\right.$ at P.A. $\left.=145^{\circ}\right)$. The continuum image shows a bright, marginally resolved source with a peak intensity of $\sim 68 \mathrm{mJy}$ beam $^{-1}$ and integrated flux density of $79 \mathrm{mJy}$. The rms noise level is $70 \mu \mathrm{Jy}_{\text {beam }}{ }^{-1}$ (the peak $\mathrm{S} / \mathrm{N}$ is $\sim 1000$; the map sensitivity is clearly limited by dynamic range). All of this emission is expected to be from dust; the periapse continuum enhancement noted by Salter et al. (2010) is not present at the observed orbital phase $(\phi=0.71)$. However, the focus here is on the emission from the $\mathrm{CO}$ spectral lines.

The ${ }^{12} \mathrm{CO}$ (hereafter $\mathrm{CO}$ ) and ${ }^{13} \mathrm{CO} J=2-1$ transitions were imaged in $0.1 \mathrm{~km} \mathrm{~s}^{-1}$-wide channels, and reach an rms noise level of $8 \mathrm{mJy} \mathrm{beam}^{-1}$ in each channel. Line emission from these transitions is detected over a $\sim 7 \mathrm{~km} \mathrm{~s}^{-1}$-wide velocity range, exhibiting the classical morphological pattern of Keplerian rotation. The peak $\mathrm{S} / \mathrm{N}$ is 35 for $\mathrm{CO}$, but only 7 for ${ }^{13} \mathrm{CO}$. Both of these transitions show considerable contamination from the local molecular cloud material, affecting a $2 \mathrm{~km} \mathrm{~s}^{-1}$-wide span slightly blueshifted from the systemic velocity. The $\mathrm{C}^{18} \mathrm{O} J=2-1$ transition was imaged in $0.2 \mathrm{~km} \mathrm{~s}^{-1}$-wide channels, with an rms of $\sim 4 \mathrm{mJy} \mathrm{beam}^{-1}$ in each, but the line is only marginally detected $(\mathrm{S} / \mathrm{N} \sim 3)$ in a few of these channels. Given the line intensities, our focus will be on an interpretation of the $\mathrm{CO}$ emission. The $\mathrm{CO}$ channel maps are shown in the top portion of Figure 1.

\subsection{Optical Spectroscopy}

Three sets of optical spectroscopic observations, including material also used by Mathieu et al. (1997), were used to reexamine the orbital solution of the DQ Tau binary. The first set consists of 30 spectra obtained at the Harvard-Smithsonian Center for Astrophysics (CfA) between 1984 and 2005 with two similar instruments equipped with intensified photon- counting Reticon detectors, as described in more detail by Mathieu et al. These spectrographs are no longer in operation. A subset of 23 of these spectra was used by Mathieu et al. (1997); we have reanalyzed all 30 of them here with improved techniques. These single-order spectra ( $45 \AA$ centered around the $\mathrm{Mg}$ Ib triplet near $5190 \AA$ ) have relatively low $\mathrm{S} / \mathrm{N}$, ranging from 6 to 16 per $8.5 \mathrm{~km} \mathrm{~s}^{-1}$ resolution element. A second set of 22 observations consists of RV differences measured from spectra also described by Mathieu et al. (1997) and collected with instruments at the Lick Observatory, the Keck Observatory, and the McDonald Observatory. Finally, more recently (2013 October to December), we obtained three additional spectra of DQ Tau at the CfA for a different purpose, with the $1.5 \mathrm{~m}$ Tillinghast reflector at the Fred L. Whipple Observatory on Mount Hopkins (AZ). For this, we used the bench-mounted TRES instrument (Fûrész 2008) that delivers a resolving power of $R \approx 44,000$ in 51 echelle orders spanning the wavelength range of $3900-9100 \AA$. These three spectra have signal-to-noise ratios in the $\mathrm{Mg} \mathrm{I} b$ region of 16,26 , and 23 per $6.8 \mathrm{~km} \mathrm{~s}^{-1}$ resolution element.

RVs for each component of DQ Tau were measured from all of the CfA spectra using the two-dimensional cross-correlation technique TODCOR (Zucker \& Mazeh 1994), with templates taken from a library of synthetic spectra based on PHOENIX model atmospheres (see Husser et al. 2013) computed for the appropriate instrumental resolution. Based on indications from the work of Mathieu et al. (1997) that the mass ratio is close to unity, we assumed the stars have the same temperature. Adopting solar metallicity, we experimented with templates of fixed surface gravities from $\log g=3.5$ to 4.5 . The best matches to the DQ Tau spectra were found for temperatures of $\sim 4000 \mathrm{~K}$ (although a relatively wide range of $\pm 300 \mathrm{~K}$ around that value is permissible) and $v \sin i$ values of 14 and $11 \mathrm{~km} \mathrm{~s}^{-1}$ for the primary and secondary, respectively. These latter values are similar to the measurements of Nguyen et al. (2012), who obtained $14.7 \pm 1.6$ and $11.3 \pm 0.7 \mathrm{~km} \mathrm{~s}^{-1}$.

The nominal temperature corresponds formally to a spectral type of $\mathrm{K} 7$, although $T_{\text {eff }}$ is degenerate with the surface gravity in our procedure because of the short wavelength coverage of the Reticon spectra from which we made these determinations. However, the RVs are unaffected by this degeneracy so long as the temperature for the templates is chosen to provide the optimal match to the spectra for a given $\log g$ value. Rotational broadening has a much larger effect on the velocities in this case because of the heavy line blending, and we believe our fine-tuning of this parameter for both stars is the reason we are able to derive meaningful velocities from all 23 of the spectra used by Mathieu et al. (1997; in addition to the other 10 from CfA used here). Their procedures only allowed them to derive separate velocities for 14 of their least blended spectra, the rest providing only an upper limit of $18 \mathrm{~km} \mathrm{~s}^{-1}$ on the velocity separation between the primary and secondary. We list our new RV measurements from all CfA spectra in Table 1.

The velocity zero point of the Reticon observations was monitored each night by means of dusk and dawn exposures of the twilight sky, and small run-to-run corrections were applied in the manner described by Latham (1992). For TRES, the zero point was monitored by observing IAU velocity standards each night. All velocities were placed on the same system, and the measurements listed in Table 1 include all corrections. 

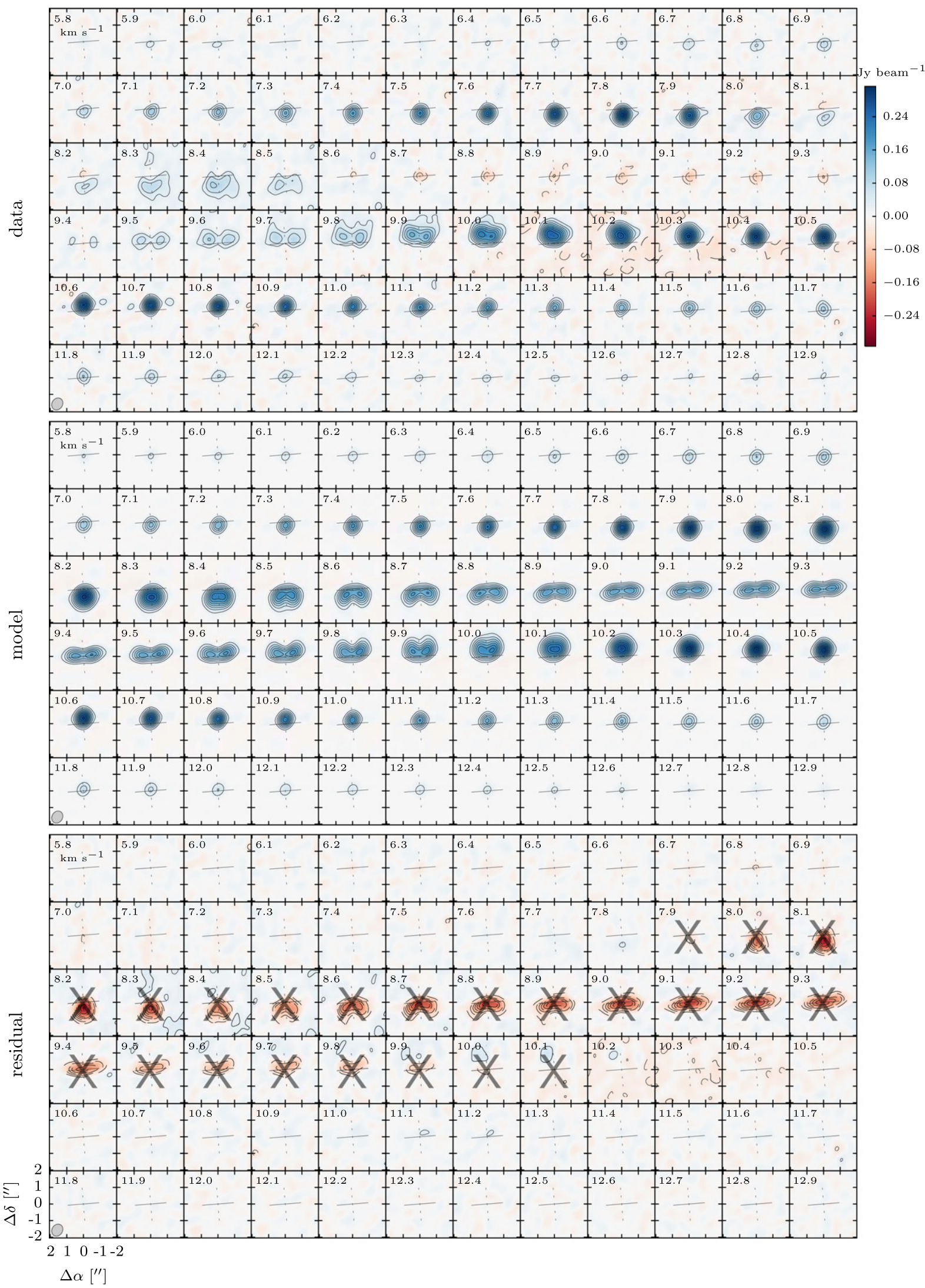

Figure 1. CO $J=2-1$ channel maps for the DQ Tau data (top), the best-fit model (middle), and the imaged residuals (bottom) at $0.1 \mathrm{~km} \mathrm{~s}{ }^{-1}$ velocity resolution. Contours are drawn at intervals of three times the rms noise level $\left(9.5 \mathrm{mJy} \mathrm{beam}^{-1}\right)$. The synthesized beam is drawn in the lower left corner of each set of channel maps, and the LSRK velocities are labeled in each panel. We do not model the channels with $v=7.9-10.1 \mathrm{~km} \mathrm{~s}^{-1}$ (inclusive) because of cloud contamination; these channel maps are marked with an $\mathrm{X}$ in the lower panel. 
Table 1

Heliocentric Radial Velocity Measurements of DQ Tau from CfA

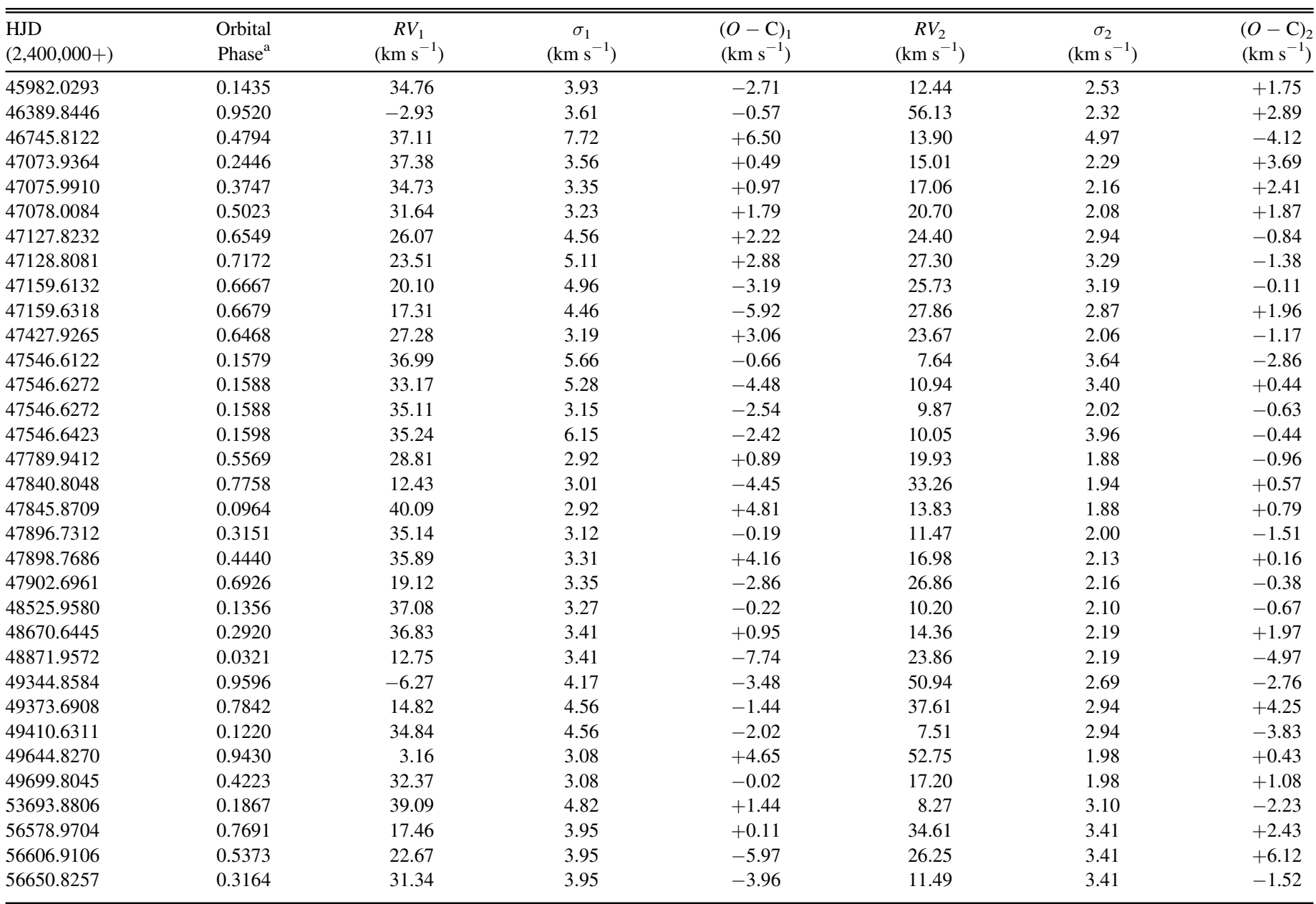

Note.

${ }^{\text {a }}$ Computed from the ephemeris given in Table 3.

\section{ANALYSIS AND RESULTS}

\subsection{CO Disk Modeling}

We analyze the CO $J=2-1$ line emission using the framework detailed in Czekala et al. (2015) and Rosenfeld et al. (2012). Briefly, we forward-model the ALMA visibilities using a parametric description of the disk structure (densities, temperatures, and velocities). For any set of model parameters, we calculate the excitation conditions in the disk assuming local thermodynamic equilibrium. We then use the RADMC-3D radiative transfer code (v0.38; Dullemond 2012) to ray-trace spectral images, which are Fourier transformed and sampled at the same spatial frequencies as the data. A $\chi^{2}$ likelihood function is used to assess the fit quality. The posterior parameter-space is explored with a Markov Chain Monte Carlo (MCMC) algorithm. ${ }^{5}$

The model parameters can be cataloged into four groups. The first group includes parameters that describe the $\mathrm{CO}$ gas densities. We assume that the standard Lynden-Bell \& Pringle (1974) similarity solution describes the radial surface density

\footnotetext{
5 The code used to perform the analysis described here is open source and freely available under an MIT license at https://github.com/iancze/ JudithExcalibur.
}

profile of the gas, which is described by an index $p,{ }^{6}$ a characteristic radius $r_{c}$, and a normalization that we cast in terms of the $\mathrm{CO}$ gas mass $M_{\mathrm{CO}}$. For computational expediency, we fix $p=1$. The second group describes the gas temperatures. We simplify the scenario by assuming a vertically isothermal structure, with a radial power-law temperature profile that has normalization $T_{10}$ (the temperature at $10 \mathrm{au}$ ) and index $q$. This thermal structure is employed in calculating the vertical density distribution, assuming hydrostatic equilibrium. The third group of parameters sets the projected velocity field of the gas, presumed to be in Keplerian rotation. It includes the central binary mass $M_{*}$, the disk inclination $i_{d}$ and position angle $\varphi$, and a systemic velocity $v_{\text {sys. }}$. Nonthermal line broadening is permitted with a line-width $\xi$ added in quadrature to the normal thermal contribution. In our convention, $i_{d}=0^{\circ}$ is a face-on disk with the angular momentum vector pointed toward the observer, $i_{d}=90^{\circ}$ is edge-on, and $i_{d}=180^{\circ}$ is face-on but with the disk angular momentum vector pointed away from the observer. The position angle $\varphi$ is defined by the projection of the angular momentum axis onto the sky. The fourth group of

\footnotetext{
6 This is more commonly $\gamma$, but we aim to avoid confusion with the standard terminology in the RV analysis (see Section 3.2).
} 
Table 2

Inferred Parameters for DQ Tau

\begin{tabular}{lccc}
\hline \hline Parameter & Value & Parameter & Value \\
\hline$T_{10}(\mathrm{~K})$ & $121 \pm 10$ & $i_{d}\left(^{\circ}\right)$ & $160 \pm 3$ \\
$q$ & $0.71 \pm 0.02$ & $\varphi\left(^{\circ}\right)$ & $94.2 \pm 0.5$ \\
$\log M_{\mathrm{CO}}\left(M_{\odot}\right)$ & $-8.0 \pm 0.3$ & $v_{\text {sys }}\left(\mathrm{km} \mathrm{s}^{-1}\right)$ & $+9.24 \pm 0.01$ \\
$r_{c}(\mathrm{AU})$ & $28 \pm 4$ & $\Delta_{\alpha}\left({ }^{\prime \prime}\right)$ & $-0.088 \pm 0.003$ \\
$M_{*}\left(M_{\odot}\right)$ & $1.27_{-0.27}^{+0.46}$ & $\Delta_{\delta}\left({ }^{\prime \prime}\right)$ & $-0.246 \pm 0.003$ \\
$\xi\left(\mathrm{km} \mathrm{s}^{-1}\right)$ & $0.18 \pm 0.02$ & $d(\mathrm{pc})$ & $155 \pm 15$ \\
\hline
\end{tabular}

Note. The quoted uncertainties represent the maximum likelihood estimate and the $68.3 \%$ highest density interval computed around this value. The systemic velocity is given in the LSRK frame for the standard radio definition, and corresponds to $+21.95 \pm 0.01 \mathrm{~km} \mathrm{~s}^{-1}$ in the barycentric frame. Samples from the posterior are published at https://figshare.com/articles/MCMC_Samples/ 2063424.

parameters is utilitarian, including the distance $d$ and nuisance offsets from the observed phase center $\left(\Delta_{\alpha}, \Delta_{\delta}\right)$.

We explore the 12-dimensional posterior-space with an ensemble MCMC sampler (Foreman-Mackey et al. 2013), employing uniform (uninformative) priors on all parameters except for $i_{d}$ and $d$. We adopt a standard geometrical prior on the disk inclination, $p\left(i_{d}\right)=\sin \left(i_{d}\right) / 2$, reflecting that there are more disk orientations that result in edge-on than face-on viewing angles. ${ }^{7}$ We choose a conservative Gaussian prior on the distance with a mean of $145 \mathrm{pc}$ and a width $(\sigma)$ of $20 \mathrm{pc}$, meant to represent the range of possible distances to sources in the Taurus clouds (e.g., Torres et al. 2010). In this analysis, we also conservatively exclude from the likelihood calculations 23 spectral channels that show evidence of molecular cloud contamination; these are marked in Figure 1. The resulting inferences on the model parameters are listed in Table 2. A comparison of the data with the model is shown in the form of channel maps in Figure 1. As was demonstrated clearly in previous work (e.g., Simon et al. 2000; Rosenfeld et al. 2012), the density- and temperature-related parameters have negligible impact on an inference of the host mass. The key parameters are $M_{*}$ and $i_{d}$ : the $\left\{M_{*}, i_{d}\right\}$ joint posterior distribution is shown in Figure 2.

We infer a mass of $1.27_{-0.27}^{+0.46} M_{\odot}$ for the DQ Tau binary, marginalized over the uncertainty contained in our distance prior. This can be expressed in a distance-independent manner as $M_{*} / d=0.0086_{-0.0018}^{+0.0021} M_{\odot} \mathrm{pc}^{-1}$; the formal uncertainty on $M_{*}$ is $\sim 25 \%$ if the distance is known exactly. This precision is significantly poorer than for most disk-based dynamical mass measurements, due to the unfortunate combination of a relatively face-on viewing geometry $\left(i_{d} \approx 160^{\circ}\right)$ and the cloud contamination around the systemic velocity.

\subsection{An Updated Spectroscopic Orbital Solution}

The orbital solution by Mathieu et al. (1997) used their 14 pairs of CfA RVs along with the velocities measured from the Lick, Keck, and McDonald observatories (hereafter the "LKM" set). Because of difficulties in maintaining a consistent velocity zero point from night to night and instrument to instrument, the

\footnotetext{
7 As we show below, the disk plane is near the plane of the sky. Given that fact and the substantial cloud contamination near the systemic velocity, we cannot uniquely determine the direction of the angular momentum axis (i.e., the sense of $i_{d}$, whether it is $\sim 160^{\circ}$ or $\sim 20^{\circ}$ ) from the ALMA data alone Therefore, we employ the astrometric constraint made by Boden et al. (2009) from infrared interferometry measurements to enforce $i>90^{\circ}$ in our analysis.
}
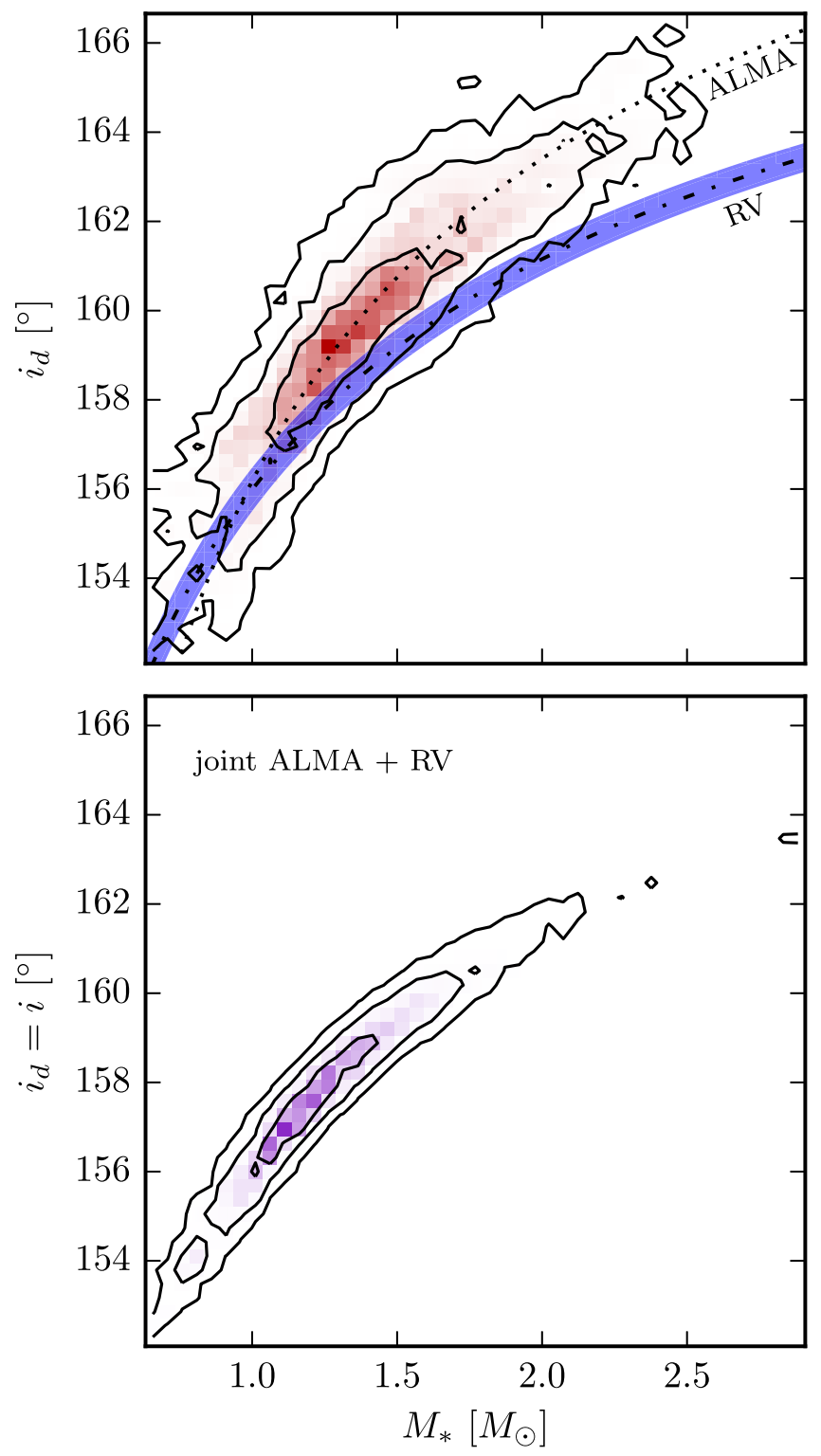

Figure 2. Top: the joint posterior distribution for $\left\{M_{*}, i_{d}\right\}$, marginalized over all other parameters. To compare with the constraints from the updated binary orbit from RV monitoring measurements, we overlay $( \pm 1 \sigma)$ contours for the measurement of $M_{*} \sin ^{3} i$ (see Section 3.2). Bottom: the joint posterior distribution combining the RV and disk measurements and assuming $i=i_{d}$. Contours denote $1 \sigma, 2 \sigma$, and $3 \sigma$ levels.

latter data were originally derived only as velocity differences between the primary and secondary, rather than individual velocities for each star. To incorporate these LKM data into a conventional double-lined orbital solution, and at the same time to tie those observations to the CfA zero point, Mathieu et al. (1997) constructed primary and secondary "measurements" from each velocity difference. They did this assuming a fixed mass ratio (of unity) and center-of-mass velocity, based on the values inferred from an initial fit based only on their 14 CfA velocity pairs (where they found $M_{2} / M_{1}=0.97 \pm 0.15$ and $\gamma=22 \mathrm{~km} \mathrm{~s}^{-1}$ ). They then combined all of the measurements into a final fit, but were careful to note that both $\gamma$ and the velocity semiamplitudes $K_{1}$ and $K_{2}$ are biased and should be ignored in favor of the values from the CfA-only solution, and similarly with the minimum masses and projected semimajor axes, which depend on the semiamplitudes. 
Table 3

Updated Spectroscopic Orbital Solution for DQ Tau

\begin{tabular}{lc}
\hline \hline Parameter & Value \\
\hline$P($ days $)$ & $15.80158 \pm 0.00066$ \\
$\gamma\left(\mathrm{km} \mathrm{s}^{-1}\right)$ & $+24.52 \pm 0.33$ \\
$K_{1}\left(\mathrm{~km} \mathrm{~s}^{-1}\right)$ & $20.28 \pm 0.71$ \\
$K_{2}\left(\mathrm{~km} \mathrm{~s}^{-1}\right)$ & $21.66 \pm 0.60$ \\
$e$ & $0.568 \pm 0.013$ \\
$\omega_{1}(\mathrm{deg})$ & $231.9 \pm 1.8$ \\
$T_{\text {peri }}(\mathrm{HJD}-2,400,000)$ & $47433.507 \pm 0.094$ \\
$M_{1} \sin ^{3} i\left(M_{\odot}\right)$ & $0.0348 \pm 0.0017$ \\
$M_{2} \sin ^{3} i\left(M_{\odot}\right)$ & $0.0326 \pm 0.0020$ \\
$\left(M_{1}+M_{2}\right) \sin ^{3} i\left(M_{\odot}\right)$ & $0.0674 \pm 0.0033$ \\
$a_{1} \sin ^{6}\left(10^{6} \mathrm{~km}\right)$ & $3.63 \pm 0.13$ \\
$a_{2} \sin i\left(10^{6} \mathrm{~km}\right)$ & $3.87 \pm 0.11$ \\
$a \sin i\left(R_{\odot}\right)$ & $10.78 \pm 0.18$ \\
$q \equiv M_{2} / M_{1}$ & $0.936 \pm 0.051$ \\
$\sigma_{1}\left(\mathrm{~km} \mathrm{~s}^{-1}\right)$ & 3.44 \\
$\sigma_{2}\left(\mathrm{~km} \mathrm{~s}^{-1}\right)$ & 2.26 \\
$\sigma_{\mathrm{LKM}}\left(\mathrm{km} \mathrm{s}^{-1}\right)$ & 2.50 \\
Time span (days) & 10668.8 \\
Time span (orbital cycles) & 675.2 \\
$N_{\mathrm{RV}}$ & $33 \times 2$ \\
$N_{\mathrm{LKM}}$ & 22 \\
\hline
\end{tabular}

Note. These results are based on a joint fit of the individual primary/secondary velocities from CfA and the LKM velocity differences. The physical constants used here are those adopted by Torres et al. (2010), consistent with the 2015 IAU Resolution B3.

For this work, we have preferred to incorporate the LKM velocity differences directly into the fit in their original form. We therefore reconstructed the original velocity differences trivially from the primary and secondary "measurements" reported by Mathieu et al. (1997) in their Table 1. The $22 \mathrm{RV}$ differences were combined in a weighted least-squares fit with our 33 pairs of primary/secondary velocities, yielding the elements listed in Table 3. For the individual velocities, weights were calculated from the internal errors. The LKM velocity differences were assigned reasonable nominal errors to begin with, and all uncertainties were then adjusted iteratively so as to obtain reduced $\chi^{2}$ values near unity for each type of measurement (primary, secondary, RV difference). Final rootmean-square residuals, which are representative of the typical measurement errors, are given in the table. The global fit derives most of the constraint on the mass ratio from the individual primary and secondary velocities. The RV differences strongly constrain the $K_{1}+K_{2}$ sum, but they also help to strengthen the individual $K$ values indirectly to some extent through constraints on the remaining orbital elements. We initially allowed for a difference in the center-of-mass velocities for the primary and secondary, to account for possible biases in the RVs that may occur as a result of template mismatch, but found the difference to be insignificantly different from zero $\left(-0.61 \pm 0.71 \mathrm{~km} \mathrm{~s}^{-1}\right)$. Consequently, the final fit assumed a common value of $\gamma$.

A graphical representation of the 33 pairs of primary/ secondary velocities from CfA is presented in Figure 3, along with our best-fit model from the global fit that includes the LKM velocity differences. In Figure 4, we illustrate the good agreement between the same best-fit model (solid curve) and the LKM velocity differences. The deviations between this best-fit model and a separate one that uses only the 33

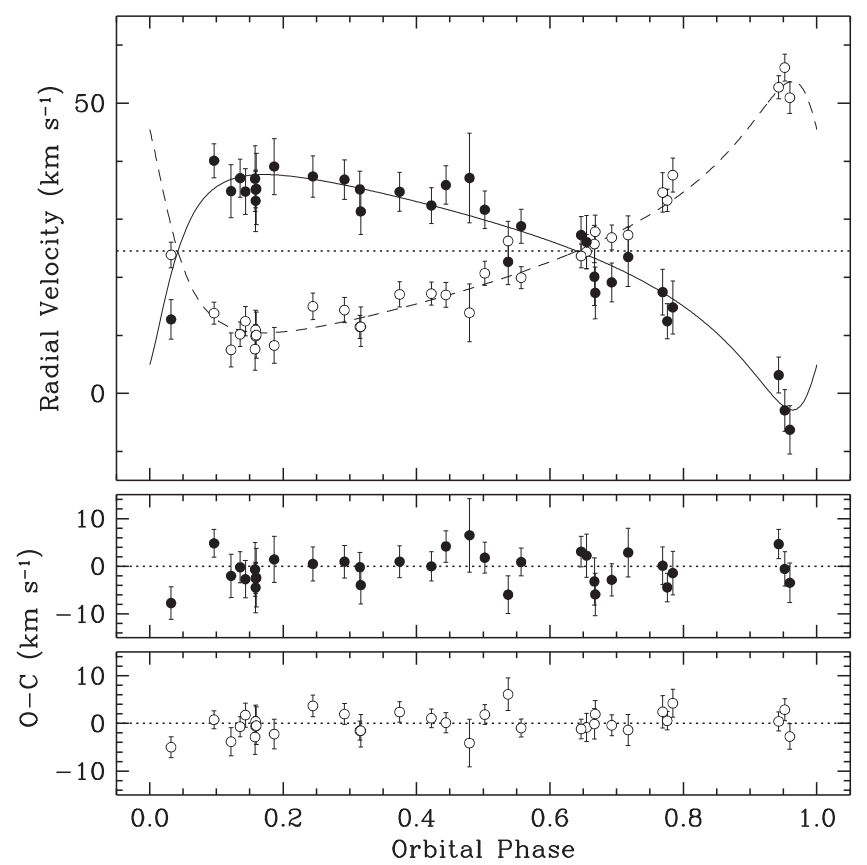

Figure 3. CfA radial-velocity measurements of DQ Tau as a function of orbital phase, including our best-fit model that also uses the LKM velocity differences. Primary velocities are represented with filled symbols, and the dotted line marks the center-of-mass velocity. The bottom panels show the residuals.

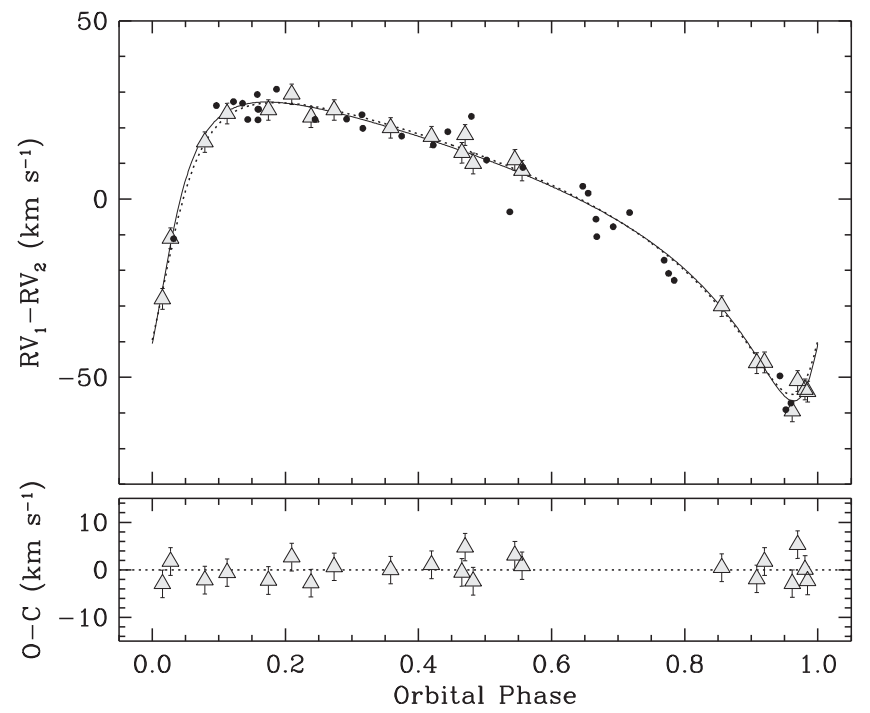

Figure 4. Predicted velocity differences as a function of orbital phase according to our best-fit model (solid line), shown with the measured LKM values (triangles). Residuals are displayed at the bottom. Also shown for reference in the top panel is an orbit model that uses only the 33 individual RV measurements from CfA (dotted line), which is nearly indistinguishable from the global fit. The dots represent the velocity differences we compute from the CfA measurements, to show that both types of measurements are fully consistent with each other.

individual CfA velocities (dotted line in the figure) are minimal.

Our results in Table 3 are generally consistent with those of Mathieu et al. (1997), but with uncertainties typically reduced by factors of two to five. The minimum masses now have relative uncertainties of $5 \%-6 \%$ instead of $\sim 21 \%$. 


\section{DISCUSSION.}

We have reported a new dynamical constraint on the mass of the young DQ Tau binary made by reconstructing the velocity field of its circumbinary disk using ALMA observations of its $\mathrm{CO}$ line emission, as well as an update on the binary orbital parameters based on a long-term optical spectroscopic monitoring campaign. In the following sections, we compare these constraints in more detail and discuss them in the context of pre-MS evolutionary model predictions and similar measurements for other equal-mass binary systems.

\subsection{Comparison of Disk- and Binary-based Constraints}

The disk-based dynamical mass approach formally constrains the quantity $\left(M_{*} / d\right) \sin ^{2} i_{d}$ by reconstructing the skyprojected Keplerian velocity field of the gas disk. Given some prior information on the distance and sufficient resolution to determine the aspect ratio of the emission $\left(i_{d}\right)$, a precise estimate of $M_{*}$ can be made. This is not quite the case for DQ Tau. The disk orbital plane is oriented such that it is viewed nearly in the plane of the sky, which concentrates most of the more spatially extended molecular line emission near the systemic velocity. This would be fine given our ALMA observations, except for the ambient molecular cloud material that also produces extended emission at those same velocities. Taken together, the small projection factor and severe cloud contamination significantly expand the $\left\{M_{*}, \sin ^{2} i_{d}\right\}$ degeneracy (see Figure 2), limiting the precision of our dynamical mass constraint. For a conservative prior on $d$, we measure a joint constraint of $M_{*} \sin ^{2} i_{d}=0.164 \pm 0.016 M_{\odot}$, or individual measurements of $1.27_{-0.27}^{+0.46} M_{\odot}$ and $i_{d}=160 \pm 3^{\circ}$.

The orbital solution for a double-lined spectroscopic binary determines $M_{*} \sin ^{3} i$ (independent of $d$ ) from a fit to a time series of RV measurements. The updated solution presented here has $M_{*} \sin ^{3} i=0.0674 \pm 0.0033 M_{\odot}$. Figure 2 confirms that the ALMA disk-based and RV binary-based constraints are in good agreement (well within $1 \sigma$ ) in the binary massinclination plane. This suggests that these constraints can be combined together to yield some informative combined measurements for the system. If we assume that the binary and disk orbital planes are exactly aligned $\left(i=i_{d}\right)$, the joint constraints from the RV and ALMA data indicate $M_{*}=1.21 \pm 0.26 M_{\odot}$ and $i=158 \pm 2^{\circ}$ (this composite posterior is shown in the bottom panel of Figure 2). If we consider the ALMA constraint on the quantity $M_{*} / d$ rather than marginalizing over the prior on $d$, we can use the assumption of coplanarity and the RV data to estimate a dynamical distance to the system. In that case, we estimate $d_{\mathrm{dyn}}=184 \pm 26 \mathrm{pc}$, which, though imprecise, has a most probable value slightly higher (at the $\sim 1 \sigma$ level) than standard measurements for the Taurus star-forming region (e.g., Torres et al. 2010) and our adopted prior, which may suggest a larger depth of the Taurus complex. In the context of our nominal prior on $d$, we can also use both data sets to instead infer a limit on the mutual inclination angle between the disk and binary orbital planes: we find that $\psi \equiv i-i_{d}=-1.3 \pm 1.1$. Interestingly, we note a small discrepancy between the systemic velocity in the barycentric frame derived from the disk $\left(+21.95 \pm 0.01 \mathrm{~km} \mathrm{~s}^{-1}\right)$ and that derived from the binary orbit $\left(+24.52 \pm 0.33 \mathrm{~km} \mathrm{~s}^{-1}\right)$. We speculate that this offset may be caused by veiling of the stellar photospheres, which results in a sub-optimal fit of the spectroscopic templates used for the RV determinations.

\subsection{Comparison to Pre-MS Evolution Models}

Having demonstrated that independent dynamical constraints on $M_{*}$ for the DQ Tau binary yield consistent results, it is of interest to make a comparison with the more common approach of estimating masses (and ages) from theoretical pre-MS evolutionary models.

A range of (combined-light) spectral types has been reported for DQ Tau, with a general consensus around M0-M1. Individual spectral diagnostics often skew toward earlier or later spectral types: for example, Basri et al. (1997) found that ratios of temperature sensitive $\mathrm{Sc}_{\mathrm{I}}$ lines suggest a $\mathrm{K} 4-\mathrm{K} 5$ classification, while Bary \& Petersen (2014) showed that many infrared molecular features (e.g., $\mathrm{TiO}, \mathrm{FeH}$, and $\mathrm{H}_{2} \mathrm{O}$ ) are better matched with a later type, M2.5-M4.5. Some of this ambiguity may be due to the implicit assumption that both stars have identical photospheric properties. The improved orbital solution in Section 3.2 suggests otherwise: the inferred mass ratio $\left(M_{2} / M_{1}=0.93 \pm 0.05\right)$ indicates that the DQ Tau stars have different temperatures and luminosities.

With that in mind, we explored a two-component fit to the BVRIJ photometry compiled by Rydgren (1984), Kolotilov (1989), and Skrutskie et al. (2006) (previously presented by Andrews et al. 2013). Observations in the $U$-band and at longer infrared wavelengths were excluded due to contamination by accretion activity and dust emission, respectively. The adopted model magnitudes were interpolated for a given $\left\{T_{\text {eff }}, \log g\right\}$ from the BT-SETTL synthetic photometry catalog (Allard et al. 2003) for solar metallicity. These were adjusted for extinction using the Fitzpatrick (1999) reddening law (with $R_{V}=3.1$ ) and scaled to account for a given luminosity (assuming the same prior on $d$ as in Section 3.1). After some experimentation, we found that the effects of surface gravity are relatively small (given the other uncertainties), so we fixed $\log g=4.0$ for both stars. Each model, therefore, has five physical parameters, $\left\{T_{1}, L_{1}, T_{2}, L_{2}, A_{V}\right\}$. We used an additional five nuisance parameters (one per band) to describe the "jitter" (dispersion) in each photometric band due to variability (presumed to be described by a Gaussian with mean zero and this parametric description of the variance). The model quality for a given set of parameters was determined with a $\chi^{2}$ likelihood function and a reasonable set of priors. At each posterior draw, we calculated the implied mass ratio and imposed a Gaussian prior with mean 0.93 and dispersion 0.05 , based on the RV orbital solution. Since photometry alone is a poor diagnostic of $T_{\text {eff }}$ (especially for a composite data set), we adopted a Gaussian prior with mean of $3900 \mathrm{~K}$ and dispersion of $250 \mathrm{~K}$ on $T_{1}$ and $T_{2}$ and enforced the conditions $T_{2} \leqslant T_{1}$ and $L_{2} \leqslant L_{1}$.

Figure 5 provides a summary of the modeling results. We find that the photometry prefers relatively low temperatures, $T_{1}=3700 \pm 200 \mathrm{~K}$ and $T_{2}=3500 \pm 175 \mathrm{~K}$, and extinction, $A_{V}=0.5 \pm 0.2$, yielding logarithmic luminosities $\log _{10} L / L_{\odot}$ of $-0.73 \pm 0.16$ and $-0.87 \pm 0.16$ for the primary and secondary, respectively. These are not particularly stringent constraints on the binary location in the HR diagram, of course, owing to the relatively ambiguous spectral classifications available for the individual components.

Based on the joint posterior distribution of $\left\{T_{1}, L_{1}, T_{2}, L_{2}\right\}$, we followed the formalism of Jørgensen \& Lindegren (2005) to 

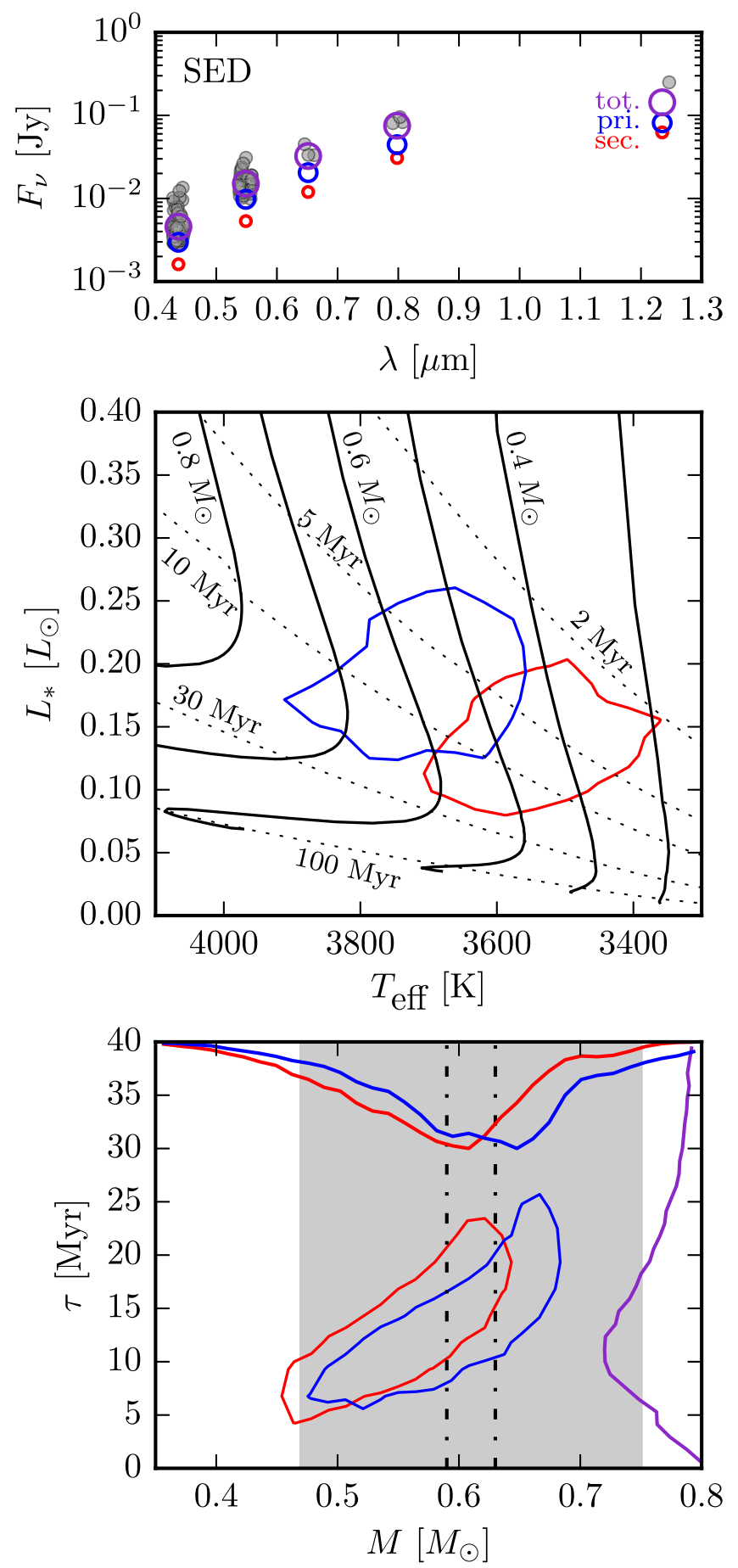

Figure 5. Top: the best-fit models of the broadband photometry overlaid on the data. Middle: the resulting HR diagram, with the marginal posteriors inferred from the photometry modeling shown as $1 \sigma$ contours. The Dotter et al. (2008) pre-MS model mass tracks and isochrones are overlaid. Bottom: the joint mass and age constraints from the Dotter et al. (2008) pre-MS models assuming the stars are coeval, shown as $1 \sigma$ contours. The marginalized distributions are shown at the boundaries of the plot. The gray band marks the disk-based constraint on the individual component mass $M_{*}(1 \sigma)$. Other pre-MS model predictions give generally comparable results.

derive component masses and ages $\left\{\tau, M_{1}, M_{2}\right\}$ from the predictions of pre-MS evolutionary models in the HR diagram, assuming that the binary stars are coeval. Various incarnations of such models (Siess et al. 2000; Dotter et al. 2008; Tognelli et al. 2011; Baraffe et al. 2015) make consistent predictions within the (considerable) uncertainties, indicating a total binary mass of $1.20 \pm 0.16 M_{\odot}$ that is in good agreement with the dynamical constraints from the ALMA and RV data. The corresponding age predictions are considerably more uncertain; favored values are in the 6-10 Myr range, although the permissible ages span from $\sim 6$ to $20 \mathrm{Myr}(1 \sigma)$. We note that this analysis is under the assumption of coevality of the two stars, which may not necessarily be true. Additionally, the unusual nature of the DQ Tau system (e.g., colliding magnetospheres during periastron) may also invalidate our assumptions of normal pre-MS evolution.

\section{SUMMARY AND CONTEXT}

We have presented a set of new constraints on the fundamental properties of the DQ Tau young binary system, based on ALMA observations of molecular line emission from its circumbinary disk and an updated analysis of optical spectroscopic measurements of its (stellar) RV variations. For a conservative distance prior $(d=145 \pm 20 \mathrm{pc})$, we find that the disk-based and binary-based dynamical constraints on the total stellar mass in the DQ Tau system are in excellent agreement: their combined inputs suggest a total mass of $M_{*}=1.21 \pm 0.26 M_{\odot}$, and therefore individual component masses $M_{1}=0.63 \pm 0.13 M_{\odot}$ and $M_{2}=0.59 \pm 0.13 M_{\odot}$ (incorporating the uncertainty on $q$ ). Moreover, we also find that the disk and binary orbital planes are aligned within $3^{\circ}$, showing that the system is coplanar across radial distances from $\sim 0.1$ to $100 \mathrm{AU}$. In this system, the dynamical mass precision is limited by an unfortunate combination of two factors: an orbital plane that is oriented nearly in the sky plane, and some large-scale contamination of the disk $\mathrm{CO}$ spectral emission from the ambient molecular cloud. In the future, an accurate parallax from GAIA will help improve the precision of the disk-based estimate of $M_{*}$.

We also estimated the stellar mass in the system using the more common technique that compares the component locations in the HR diagram with predictions of theoretical pre-MS evolution models, and generally found good agreement. However, that approach has restricted utility given the lack of component-resolved photometry and substantial ambiguity on the effective temperatures. There is still much to be learned from this fascinating system; our mass constraints lend some quantitative benchmarks that can be adopted in future studies.

DQ Tau is the third nearly equal-mass young binary system that has been analyzed with these two independent dynamical techniques to constrain stellar masses, the others being the older- and earlier-type systems V4046 Sgr (total mass $1.75 M_{\odot}$; Rosenfeld et al. 2012) and AK Sco (total mass 2.50 $\mathrm{M}_{\odot}$; Czekala et al. 2015). Using millimeter-wave interferometric measurements of their $\mathrm{CO}$ spectral line emission, model fitting that reconstructs the Keplerian velocity fields of their circumbinary disks finds dynamical masses that are in excellent agreement with constraints from optical RV monitoring of the host binaries (thereby also implying that the binary and disk orbits are coplanar). Granted, this is a small sample, but it does span an important range of system properties: e.g., spectral types from early $\mathrm{M}$ to mid $\mathrm{F}$, ages from a few to tens of megayears, and orbital eccentricities from circular to $e \approx 0.6$. Taken together, this work validates the quantitative accuracy of the disk-based dynamical inference of young star masses, provided that it is done carefully in a proper analysis 
framework. Moving forward, this confirms that ALMA should play a substantial role in young star astrophysics, as the technique used here is the only means of precisely measuring the masses for large samples of single stars.

We appreciate some useful computational suggestions from Ryan Loomis. I.C. gratefully acknowledges funding support from the Smithsonian Institution. S.A. appreciates the very helpful support provided by the NRAO Student Observing Support program related to the early development of this project. This paper makes use of the following ALMA data: 2012.1.00496.S. ALMA is a partnership of ESO (representing its member states), NSF (USA), and NINS (Japan), together with NRC (Canada) and NSC and ASIAA (Taiwan), in cooperation with the Republic of Chile. The Joint ALMA Observatory is operated by ESO, AUI/NRAO, and NAOJ. Figure 2 was generated with the triangle.py code (Foreman-Mackey et al. 2014). This research made extensive use of the Julia programming language (Bezanson et al. 2012) and Astropy software package (Astropy Collaboration 2013).

\section{REFERENCES}

Allard, F., Guillot, T., Ludwig, H.-G., et al. 2003, in Proc. IAU Symp. 211, Brown Dwarfs, ed. E. Martín (San Francisco, CA: ASP), 325

Andrews, S. M., Rosenfeld, K. A., Kraus, A. L., \& Wilner, D. J. 2013, ApJ, 771,129

Andrews, S. M., \& Williams, J. P. 2005, ApJ, 631, 1134

Astropy Collaboration 2013, A\&A, 558, 33

Baraffe, I., Chabrier, G., \& Gallardo, J. 2009, ApJL, 702, L27

Baraffe, I., Homeier, D., Allard, F., \& Chabrier, G. 2015, arXiv:1503.04107

Bary, J. S., \& Petersen, M. S. 2014, ApJ, 792, 64

Basri, G., Johns-Krull, C. M., \& Mathieu, R. D. 1997, AJ, 114, 781

Beckwith, S. V. W., Sargent, A. I., Chini, R. S., \& Güsten, R. 1990, AJ, 99, 924
Bezanson, J., Karpinski, S., Shah, V. B., \& Edelman, A. 2012, arXiv: 1209.5145

Boden, A. F., Akeson, R. L., Sargent, A. I., et al. 2009, ApJL, 696, L111

Czekala, I., Andrews, S. M., Jensen, E. L. N., et al. 2015, ApJ, 806, 154

Dotter, A., Chaboyer, B., Jevremović, D., et al. 2008, ApJS, 178, 89

Dullemond, C. P. 2012, Astrophysics Source Code Library, ascl:1202.015

Feiden, G. A., \& Chaboyer, B. 2013, ApJ, 779, 183

Fitzpatrick, E. L. 1999, PASP, 111, 63

Foreman-Mackey, D., Hogg, D. W., Lang, D., \& Goodman, J. 2013, PASP, 125,306

Foreman-Mackey, D., Price-Whelan, A., Ryan, G., et al. 2014, http://dx.doi. org $/ 10.5281$ /zenodo. 11020

Fúrész, G. 2008, PhD thesis, Univ. Szeged

Guilloteau, S., Di Folco, E., Dutrey, A., et al. 2013, A\&A, 549, A92

Guilloteau, S., Dutrey, A., Piétu, V., \& Boehler, Y. 2011, A\&A, 529, A105

Herbig, G. H. 1977, ApJ, 214, 747

Herczeg, G. J., \& Hillenbrand, L. A. 2014, ApJ, 786, 97

Hillenbrand, L. A., \& White, R. J. 2004, ApJ, 604, 741

Husser, T.-O., Wende-von Berg, S., Dreizler, S., et al. 2013, A\&A, 553, A6

Jørgensen, B. R., \& Lindegren, L. 2005, A\&A, 436, 127

Kolotilov, E. A. 1989, AZh, 66, 335

Latham, D. W. 1992, in ASP Conf. Ser. 32, IAU Coll. 135, Complementary Approaches to Double and Multiple Star Research, ed. H. A. McAlister, \& W. I. Hartkopf (San Francisco, CA: ASP), 110

Lynden-Bell, D., \& Pringle, J. E. 1974, MNRAS, 168, 603

Mathieu, R. D., Stassun, K., Basri, G., et al. 1997, AJ, 113, 1841

Nguyen, D. C., Brandeker, A., van Kerkwijk, M. H., \& Jayawardhana, R. 2012, ApJ, 745, 119

Rosenfeld, K. A., Andrews, S. M., Wilner, D. J., \& Stempels, H. C. 2012, ApJ, 759, 119

Rydgren, A. E. 1984, PUSNO, 25, 1

Salter, D. M., Kóspál, Á, Getman, K. V., et al. 2010, A\&A, 521, A32

Siess, L., Dufour, E., \& Forestini, M. 2000, A\&A, 358, 593

Simon, M., Dutrey, A., \& Guilloteau, S. 2000, ApJ, 545, 1034

Skrutskie, M. F., Cutri, R. M., Stiening, R., et al. 2006, AJ, 131, 1163

Stassun, K. G., Feiden, G. A., \& Torres, G. 2014, NewAR, 60, 1

Tognelli, E., Prada Moroni, P. G., \& Degl'Innocenti, S. 2011, A\&A, 533, A109

Torres, G., Andersen, J., \& Giménez, A. 2010, A\&ARv, 18, 67

Williams, J. P., \& Best, W. M. J. 2014, ApJ, 788, 59

Zucker, S., \& Mazeh, T. 1994, ApJ, 420, 806 